\title{
Unsteady three-dimensional boundary layer flow due to a permeable shrinking sheet
}

\begin{abstract}
The unsteady viscous flow over a continuously permeable shrinking surface is studied. Similarity equations are obtained through the application of similar transformation techniques. Numerical techniques are used to solve the similarity equations for different values of the unsteadiness parameter, the mass suction parameter, the shrinking parameter and the Prandtl number on the velocity and temperature profiles as well as the skin friction coefficient and the Nusselt number. It is found that, different from an unsteady stretching sheet, dual solutions exist in a certain range of mass suction and unsteadiness parameters.
\end{abstract}

Keyword: Unsteady; Three-dimensional flow; Boundary layer; Shrinking sheet; Dual solution 